\title{
Levofloxacin versus clarithromycin in COPD exacerbation: focus on exacerbation-free interval
}

\author{
H. Lode, J. Eller, A. Linnhoff, M. Ioanas, and the Evaluation of Therapy-Free Interval in COPD Patients \\ Study Group
}

Levofloxacin versus clarithromycin in COPD exacerbation: focus on exacerbation-free interval. H. Lode, J. Eller, A. Linnhoff, M. Ioanas, and the Evaluation of Therapy-Free Interval in COPD Patients Study Group. (C) ERS Journals Ltd 2004.

ABSTRACT: Antibiotic treatment of bacterial exacerbation of chronic obstructive pulmonary disease (COPD) shows some immediate clinical benefits and may also minimise the frequency of further recurrences.

Patients $(n=511)$ were enrolled into a randomised double-blind multicentric study comparing the exacerbation-free interval (EFI), efficacy and safety of 7-day levofloxacin versus 10-day clarithromycin in patients with COPD exacerbation. Patients were monitored over a 1-yr period. A total of 434 patients (per protocol population) received the medication for $\geqslant 5$ days.

The median EFI in the per protocol population was 300 days for levofloxacin and 350 days for clarithromycin. For patients with a new documented exacerbation during follow-up ( $n=223$ ), the median EFI was 100.5 days in the levofloxacin group and 95 days for clarithromycin. No significant differences in EFI between groups could be observed when stratifying the study population according to microbial aetiology and severity of bronchial obstruction. Levofloxacin and clarithromycin showed similar clinical success rates. The bacteriological success rate was significantly higher in the levofloxacin group. Both antibiotics were well tolerated.

In summary, levofloxacin was associated with a significantly higher bacteriological eradication rate but similar exacerbation-free interval in patients with chronic obstructive pulmonary disease exacerbation compared to clarithromycin. Eur Respir J 2004; 24: 947-953.
Helios Hospital Emil von Behring, Berlin, Germany.

Correspondence: H. Lode

Helios Klinikum Emil von Behring

Zum Heckeshorn 33

D-14109 Berlin

Germany

Fax: 493080022623

E-mail: haloheck@zedat.fu-berlin.de

Keywords: Chronic obstructive pulmonary disease

clarithromycin

exacerbation

exacerbation-free interval

levofloxacin

Received: January 232004

Accepted after revision: July 162004

This study was supported by a grant from Aventis Pharma, Bad Soden, Germany.
Acute exacerbations of chronic obstructive pulmonary disease (COPD) are typical events that characterise the course of the disease and are the most common cause of death in these patients $[1,2]$. Exacerbations result in impaired quality of life, decline in lung function and increased health care utilisation [3-5]. Although the aetiology of COPD exacerbation has not been completely clarified, there is strong evidence that potentially pathogenic microorganisms (PPMs) are isolated from at least half of COPD patients during exacerbations [6-9]. Another $30-40 \%$ of the exacerbations of COPD were recently shown to be attributable to viruses $[10,11]$.

In this context, antimicrobial therapy remains a controversial issue, although it shows some immediate clinical benefits compared to no therapy [12]. A clear indication for antibiotic treatment appears to be sputum purulence, a simple parameter for discriminating between bacterial and nonbacterial exacerbation [13].

Fluoroquinolones seem to be an adequate choice, taking into account their bactericidal activity in vitro against most of the pathogens involved in COPD exacerbation, including penicillin-resistant Streptococcus pneumoniae (gatifloxacin, moxifloxacin, levofloxacin and gemifloxacin) and Pseudomonas aeruginosa (ciprofloxacin). Furthermore, the good penetration

For editorial comments see page 896. into lung tissue and respiratory secretions, one-dosage daily administration (for the new quinolones) and short duration of treatment also favour choice of these drugs in COPD exacerbation.

Several clinical trials support the clinical efficacy of fluoroquinolones in exacerbation of chronic bronchitis $[14,15]$ compared to macrolides or $\beta$-lactams. Moreover, the recent study of WILSON et al. [16] shows that fluoroquinolones (i.e. gemifloxacin) exhibit an additional benefit compared to macrolides (i.e. clarithromycin), in that they reduce the recurrence of exacerbations. Since fluoroquinolones and macrolides seem to exhibit rather comparable clinical and bacteriological efficacy, as well as similar safety profiles $[14,16]$, this finding may have considerable impact on therapeutic choice, especially in COPD patients with frequent exacerbations.

Based on these data, the aim of the present study was to compare the exacerbation-free interval (EFI) following treatment with levofloxacin and clarithromycin in COPD exacerbation. Several clinical trials have demonstrated that levofloxacin shows clinical and bacteriological efficacy in acute exacerbation of chronic bronchitis [14, 17, 18]. Clarithromycin was used as comparator because of its proven efficacy in this condition [16, 19]. Secondary objectives included comparisons of clinical and bacteriological response, as well as the safety profile of the two antibiotics. 


\section{Methods}

\section{Study design and patients}

The current prospective randomised multicentric doubleblind comparative study was performed using a doubledummy design with two-arm parallel groups. Patients were randomised in a 1:1 ratio to receive either oral levofloxacin (Aventis Pharma Deutschland, Bad Soden, Germany) $500 \mathrm{mg}$ once daily for 7 days followed by placebo (Allphamed PHARBIL Arzneimittel, Göttingen, Germany) for 3 days, or clarithromycin (Abbott, Wiesbaden, Germany) $250 \mathrm{mg}$ twice daily (the officially approved dosage of clarithromycin for this condition in Germany) for 10 days.

The study included outpatients aged $>35$ yrs with a clinical history of chronic bronchitis with $\geqslant 2$ exacerbations $\cdot \mathrm{yr}^{-1}[20]$, a forced expiratory volume in one second (FEV1) in the range $35-75 \%$ predicted [21] and presenting an acute exacerbation. The last available FEV1 measurement in the stable state within the previous 6 months was considered for the inclusion criteria. The exacerbation was defined according to Winnipeg criteria (increased dyspnoea, increased sputum volume and purulent sputum) [22], and only patients meeting Winnipeg I (all three criteria) or II (two criteria present) were enrolled.

Patients were excluded from the study if they exhibited any of the following characteristics: severe respiratory infection requiring parenteral antibiotic treatment; evidence of a new pulmonary infiltrate on chest radiography; administration of another antibiotic within $72 \mathrm{~h}$ prior to enrolment; documented evidence of severe bronchiectasis; need for concomitant antimicrobial medication; current treatment with systemic steroids at a dose of $>20 \mathrm{mg} \cdot \mathrm{day}^{-1}$ prednisone or equivalent for $>4$ weeks; AIDS or another immunosuppressive condition; a history of epilepsy or lowered seizure threshold; previous enrolment in another study within the previous 4 weeks; pregnancy or nursing; allergy to fluoroquinolones or macrolide derivatives; severe underlying diseases, such as cystic fibrosis, active tuberculosis, or suspected lung or chest malignancy; progressively fatal disease; malabsorption syndrome; hepatic disease; renal impairment (creatinine level $>2.0 \mathrm{mg} \cdot \mathrm{dL}^{-1}$ ); or alcohol or drug abuse.

All patients provided written informed consent and the study protocol was approved for all centres by the local ethics committees. The study was conducted according to the Good Clinical Practice Guidelines of the European Union and the Declaration of Helsinki.

\section{Follow-up and exacerbation-free interval assessment}

Patients were monitored over a period of $1 \mathrm{yr}$, with scheduled visits at weeks $6,18,36$ and 52 . When patients could not attend a scheduled visit, they were contacted by telephone. Patients were instructed to contact the investigator(s) responsible for the study immediately if there was any change in their health status. Diagnosis of a new exacerbation was based on the same clinical criteria as the previous. During each visit, patients were asked to report any event that required antibiotic therapy and/or hospitalisation elsewhere, in order to avoid unrecorded exacerbations.

In agreement with the studies of CHODOSH and coworkers $[15,23]$, all clinical failures during the study therapy were counted as zero EFI days. For patients with no new exacerbation during the $1-y r$ observation period, the EFI was considered to be the number of days that had elapsed between the index exacerbation and the time point of the last information available (censored data). In all other cases, the number of days that had elapsed between the onset of exacerbations was taken into account. For calculation, the onset of an exacerbation was considered the day of medical attendance.

\section{Clinical and bacteriological assessments and definitions}

Clinical signs and symptoms of acute exacerbation (cough, dyspnoea, sputum characteristics and auscultatory assessment) were noted before initiating the treatment, after 3-5 days and at day 11-14 (test of cure).

Any further exacerbation occurring during the follow-up period was evaluated based on the same criteria as the index episode. The clinical response at the end of treatment, with respect to the responder analysis, was defined as follows: success, if all infection-related symptoms disappeared or were improved to such an extent that no further antibiotic therapy was indicated; failure, if there was no improvement or deterioration of the symptoms; and unknown, if no assessment was possible.

Microbiological evaluation of the sputum samples was performed before treatment, at day 3-5 and, if possible, at the end of treatment (day 11-14). According to the criteria of the American Society for Microbiology [24], only sputa with $<10$ or 10-25 squamous oropharyngeal epithelial cells and $>25$ leukocytes per low power field (x100) were considered for culture. Culture was performed according to standard microbiological methods [25]. Susceptibility was determined by a standard disc diffusion technique recommended by the National Committee for Clinical Laboratory Standards [26]. A proven bacterial aetiology was not mandatory for study enrolment.

A satisfactory bacteriological response was defined as eradication (the baseline bacteriological pathogen was eradicated) or presumed eradication (the patient had improved clinically to such an extent that a satisfactory follow-up culture from sputum samples could not be obtained). An unsatisfactory response was recorded as persistence (the baseline causative pathogen was still present irrespective of the presence or absence of signs of infection), relapse (the absence of the baseline causative pathogen was documented but the same pathogen appeared in cultures of specimens obtained after the end of treatment) or superinfection (a new causative pathogen isolated from any site during therapy or within 3 days after treatment completion, together with clinical evidence of infection).

\section{Safety assessment}

Adverse events were evaluated in all patients that received at least one dose of the study drug (safety population). Adverse events were recorded at all visits and ranked by intensity (mild, moderate, severe and serious) and relationship to the study medication.

\section{Statistical analysis}

The sample size calculation was based on survival analysis models with a two-sided type I error probability $(\alpha)$ of 0.05 and a type II error probability $(\beta)$ of 0.20 , i.e. a power of $80 \%$. Expecting a mean duration of 4-6 months to the next exacerbation under clarithromycin and a 50\% increase in the EFI under levofloxacin and assuming, furthermore, a mean observation period of $\sim 6.5$ months per patient, a required sample size of 251 patients per treatment group was estimated. 
Survival analysis techniques were employed to calculate EFIs and the Kaplan-Meier method was used to estimate the distribution of time to failure. The Wilcoxon test and logrank test were applied to compare the survival curves for each study drug group. The latter, which places more weight on later times of failure, was used for the formal testing of the study hypothesis (superiority of levofloxacin over clarithromycin). The median and, respectively, the $95 \%$ confidence interval (CI) of the median EFI could not be calculated for some subgroups, since $<50 \%$ of the study population developed a new exacerbation over the study period; therefore, the 25 th percentile has been also estimated.

The response (clinical and bacteriological) data were analysed by centre-adjusted Cochran-Mantel-Haenszel tests. In addition, two-sided $95 \%$ CIs for the treatment differences in response rates were calculated.

Comparability of baseline characteristics was descriptively analysed using the Cochran-Mantel-Haenszel test stratified by centre (categorical parameters) or analysis of variance models with the factors treatment group and centre and the interaction term of treatment by centre (continuous parameters).

Statistical analysis was performed separately on the following populations: intention-to-treat (ITT) population, including all randomised patients; safety population, including all patients who received at least one dose of the study medication; modified-ITT (m-ITT) population, consisting of patients who received the study medication for $\geqslant 2$ days; per protocol (PP) population, consisting of patients who adhered to the study protocol and received the study medication for $\geqslant 5$ days; and PP microbiology population, including all PP population patients with at least one causative pathogen isolated at enrolment and a bacteriological evaluation at the end of treatment.

\section{Results}

\section{Baseline characteristics}

The study was conducted in 36 centres in Germany, and 511 patients with a diagnosis of acute exacerbation of COPD were enrolled. The distribution of the study population is indicated in figure 1 . As one patient refused to participate before starting treatment, a total of 510 patients were evaluable in the safety analysis (safety population). Six patients were treated for $<2$ days and were evaluated only in the safety population. A total of 504 patients $(55 \%$ male; mean age $59.7 \pm 11.1 \mathrm{yrs}$ ) with a mean FEV1 of $58.1 \pm 12.1 \%$ pred measured in the stable state were included in the m-ITT analysis. The baseline characteristics of the two treatment groups were similar (table 1). A total of 477 (93.5\%) patients received the complete 10 days' medication $(94.1 \%$ in levofloxacin group and $93.0 \%$ in clarithromycin group). The most frequent comorbid conditions in the two treatment groups were cardiovascular diseases $(35.6 \%$ of patients in levofloxacin group and $38.6 \%$ in clarithromycin group) and metabolic disorders $(18.8 \%$ in levofloxacin group and $21.7 \%$ in clarithromycin group). Nearly all patients, 250 in each group, received concomitant medication over the study period, consisting of inhaled corticosteroids $(10.3 \%$ in levofloxacin group and $9.8 \%$ in clarithromycin group), and selective $\beta_{2}$-agonists $(20.4 \%$ in levofloxacin group and $19.8 \%$ in clarithromycin group) or xanthin-including derivatives $(10.9 \%$ in levofloxacin group and $10.7 \%$ in clarithromycin group).

Drug efficacy could be evaluated in 434 patients ( $\mathrm{PP}$ population; $\mathrm{n}=223$ in levofloxacin group and $\mathrm{n}=211$ in clarithromycin group). Protocol violations such as

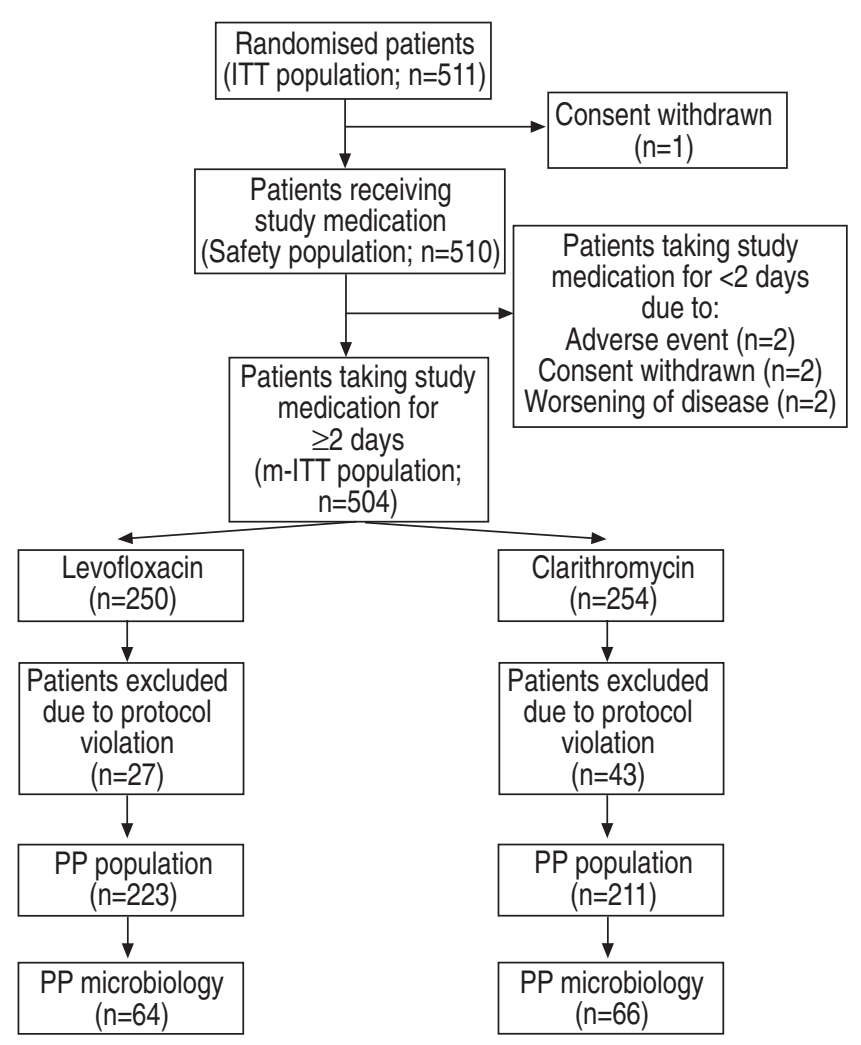

Fig. 1.-Distribution of study population. ITT: intention to treat; m-ITT: modified-ITT; PP: per protocol.

Table 1.-Baseline characteristics of the modified intentionto-treat (m-ITT) population

\begin{tabular}{|c|c|c|}
\hline & Levofloxacin & Clarithromycin \\
\hline Subjects $n$ & 250 & 254 \\
\hline Age yrs & $59.9 \pm 11.1$ & $59.6 \pm 11.1$ \\
\hline \multicolumn{3}{|l|}{ Sex } \\
\hline Male n $(\%)$ & $137(54.8)$ & $140(55.1)$ \\
\hline Female n $(\%)$ & $113(45.2)$ & $114(44.9)$ \\
\hline $\mathrm{BMI} \mathrm{kg} \cdot \mathrm{m}^{-2}$ & $27.2 \pm 5.0$ & $26.6 \pm 5.1$ \\
\hline FEV1 $\%$ pred & $57.7 \pm 11.5$ & $58.5 \pm 12.6$ \\
\hline $\begin{array}{l}\text { Time since diagnosis } \\
\text { of COPD months }\end{array}$ & $98.5 \pm 85.0$ & $108.0 \pm 90.5$ \\
\hline \multicolumn{3}{|l|}{ Smoking habit } \\
\hline Smoker n $(\%)$ & $85(34.0)$ & $83(32.7)$ \\
\hline Ex-smoker n $(\%)$ & $67(26.8)$ & $81(31.9)$ \\
\hline Nonsmoker n (\%) & $91(36.4)$ & $79(31.1)$ \\
\hline Missing data n (\%) & $7(2.8)$ & $11(4.3)$ \\
\hline \multicolumn{3}{|c|}{ Severity of bronchial obstruction } \\
\hline FEV $1 \geqslant 50 \%$ pred $\mathrm{n}(\%)$ & $183(73.2)$ & $181(71.3)$ \\
\hline FEV1 $<50 \%$ pred n $(\%)$ & $62(24.8)$ & $65(25.6)$ \\
\hline No data $\mathrm{n}(\%)$ & $5(2.4)$ & $8(3.1)$ \\
\hline \multicolumn{3}{|l|}{ Winnipeg classification ${ }^{\#}$} \\
\hline I n $(\%)$ & $181(72.4)$ & $185(72.8)$ \\
\hline II n $(\%)$ & $69(27.6)$ & $68(26.8)$ \\
\hline
\end{tabular}

Data are presented as mean \pm SD unless otherwise indicated. BMI: body mass index; FEV1: forced expiratory volume in one second; COPD: chronic obstructive pulmonary disease; \% pred: per cent predicted. \#: Winnipeg I: all three criteria present (increased dyspnoea, increased sputum volume and purulent sputum); Winnipeg II: only two criteria present.

administration of drug medication for $<5$ days $(n=24)$, violation of study timing $(n=21)$ or missing data $(n=12)$ were the primary reasons for the exclusion of 77 patients from the 
analysis of the PP population. Baseline characteristics and clinical respiratory signs and symptoms were similar in the PP population and the m-ITT group (data not shown).

\section{Exacerbation-free interval}

The EFIs are presented in table 2. No significant differences in EFI could be observed between the two study drugs in the m-ITT and PP populations. The EFI was similar in the subgroup of patients with a new documented exacerbation and in that with a documented microbial infection at enrolment.

A similar trend in the EFI was observed in the two study groups when patients were stratified according to the presence of S. pneumoniae and Haemophilus influenzae at enrolment.

A total of $43.6 \%$ of patients in the levofloxacin group and $47.9 \%$ of patients in the clarithromycin group in the m-ITT population exhibited no relapse during the 12-month period after therapy ( $\mathrm{p}=0.967)$.

When stratifying the patients according to the severity of obstruction ( $\mathrm{FEV} 1 \geqslant$ or $<50 \%$ pred), no significant differences could be observed in EFI between the two study groups (table 2) or between severity groups (data not shown).

Figure 2 shows the Kaplan-Meier survival analysis, revealing the EFIs for the m-ITT population with an FEV1 of $<50 \%$ pred.

\section{Clinical response}

In the evaluable m-ITT population $(\mathrm{n}=433)$, clinical success at the end of treatment was observed in $82.8 \%$ of patients in the levofloxacin group and $79.8 \%$ in the clarithromycin group (3.0\% difference; 95\% CI -4.4-10.3; p=0.428) (fig. 3). Regarding the evaluable PP population $(n=365)$, clinical success of 86.1 and $84.8 \%$ was documented in the levofloxacin and clarithromycin groups, respectively (1.3\% difference; $95 \%$ CI $-6.0-8.5 ; \mathrm{p}=0.732)$. In $\sim 15 \%$ of the m-ITT population and nearly $15 \%$ of the PP group, no clinical assessment was available at the end of therapy because of missing data.

\section{Bacteriological response}

PPMs responsible for the acute exacerbation were isolated in $125(50 \%)$ patients in the levofloxacin group and 131 $(51.6 \%)$ in the clarithromycin group. The most frequently isolated strains were: $H$. influenzae $(\mathrm{n}=80 ; 38(23.9 \%)$ in levofloxacin group and $42(25.8 \%)$ in clarithromycin group),

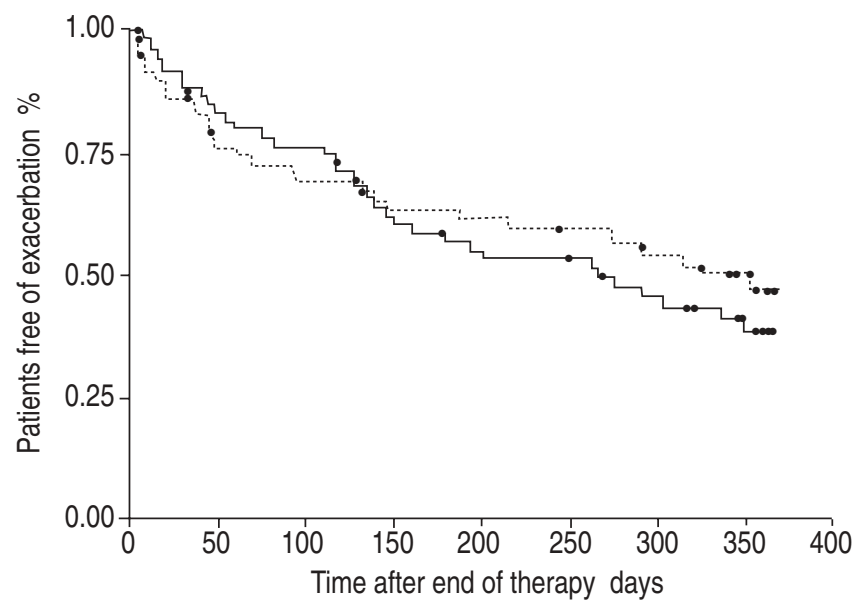

Fig. 2. - Kaplan-Meier survival analysis of exacerbation-free interval in modified intention-to-treat group patients with a forced expiratory volume in one second of $<50 \%$ predicted ( - : clarithromycin; ------: levofloxacin). No difference could be observed $(\mathrm{p}=0.322)$.

S. pneumoniae $(\mathrm{n}=50 ; 23(14.5 \%)$ in levofloxacin group and 27 $(16.6 \%)$ in clarithromycin group), Moraxella catarrhalis $(\mathrm{n}=39 ; 22(13.8 \%)$ in levofloxacin group and $17(10.4 \%)$ in clarithromycin group), and Staphylococcus aureus $(\mathrm{n}=24$; nine $(5.7 \%)$ in levofloxacin group and $15(9.2 \%)$ in clarithromycin group). Of the 322 strains of PPMs isolated at baseline, $34.5 \%$ were resistant to clarithromycin and only one strain $(S$. pneumoniae) showed an intermediate level of resistance to levofloxacin. Of the Haemophilus spp. strains, 35\% were resistant to clarithromycin, whereas none was resistant to levofloxacin.

The bacteriological eradication rate at the end of treatment in the evaluable m-ITT population with microbial aetiology $(n=251)$ was significantly higher in the levofloxacin group than in the clarithromycin group (96.0 versus $81.7 \% ; 14.3 \%$ difference; 95\% CI 6.7-21.8; $\mathrm{p}<0.0001$ ) (fig. 4). A significantly higher bacteriological success rate in favour of levofloxacin compared to clarithromycin (96.8 versus $83.1 \% ; 13.7 \%$ difference; 95\% CI 3.7-23.8; $\mathrm{p}=0.01)$ was also demonstrated in the PP microbiology group $(n=130)$.

\section{Safety and adverse events}

Forty-nine patients, $24(9.5 \%)$ in the levofloxacin group and $25(9.7 \%)$ in the clarithromycin group, reported 75 adverse events ( 31 and 44, respectively), considered to be at least

Table 2. - Exacerbation-free interval (EFI) in days

\begin{tabular}{|c|c|c|c|c|}
\hline \multirow[t]{2}{*}{ Population } & \multirow[t]{2}{*}{ Subjects $n$} & \multicolumn{2}{|c|}{ EFI days } & \multirow[t]{2}{*}{ p-value $\#$} \\
\hline & & Levofloxacin & Clarithromycin & \\
\hline m-ITT & 504 & $98(50-134) ; 324$ & $97(59-130) ; 365$ & 0.504 \\
\hline $\mathrm{PP}$ & 434 & $95(46-131) ; 300$ & $84(51-129) ; 350$ & 0.594 \\
\hline New exacerbation & 223 & $33(22-44) ; 100.5(59-134)$ & $33(30-47) ; 95(58-123)$ & 0.630 \\
\hline PPMs in sputum at baseline & 246 & $59(41-224)$ & $95(47-134)$ & 0.907 \\
\hline Haemophilus influenzae & 80 & $42(22-95) ; 131$ & 47 (19-276); 299 & 0.632 \\
\hline Streptococcus pneumoniae & 50 & $45(36-327) ; 327$ & 134 (33-264); 264 & 0.715 \\
\hline New exacerbation & 100 & $22(19-41) ; 50(40-131)$ & 32 (19-47); 76 (46-123) & 0.378 \\
\hline \multicolumn{5}{|c|}{ Severity of bronchial obstruction } \\
\hline FEV $1 \geqslant 50 \%$ pred & 362 & $107(52-145) ; 300$ & $95(51-164)$ & 0.215 \\
\hline FEV $1<50 \%$ pred & 129 & $60(37-187) ; 355$ & $110(47-146) ; 266$ & 0.509 \\
\hline
\end{tabular}

Data are presented as 25 th percentile; median with, where available, two-sided $95 \%$ confidence interval in parenthesis. m-ITT: modified intent to treat; PP: per protocol; PPMs: potentially pathogenic microorganisms; FEV1: forced expiratory volume in one second; \% pred: per cent predicted. ${ }^{\text {: }}$ log-rank test; ${ }^{\uparrow}$ : in patients with documented PPMs in sputum at enrolment. 


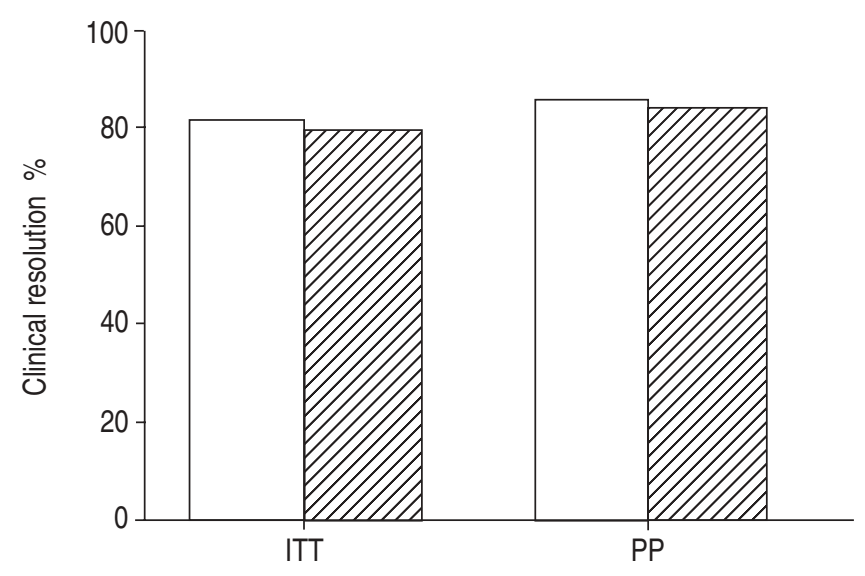

Fig. 3.-Clinical resolution at the end of therapy in the evaluable intent-to-treat (ITT) and per protocol (PP) populations of patients with chronic obstructive pulmonary disease exacerbation treated with levofloxacin $(\square)$ or clarithromycin $(\mathbb{C})$.

possibly drug related. Most frequent were gastrointestinal adverse drug reactions (5.5\% in levofloxacin group and $6.6 \%$ in clarithromycin group) and disorders of the central nervous system $(0.8 \%$ in levofloxacin group and $2.3 \%$ in clarithromycin group). Most adverse events were mild to moderate. Study drug treatment was prematurely discontinued because of one or more adverse events in 26 patients $(n=14$ in levofloxacin group and $n=12$ in clarithromycin group), mostly due to gastrointestinal symptoms.

\section{Discussion}

The present study showed no difference in EFI between treatment with levofloxacin and clarithromycin in acute exacerbation of COPD. Levofloxacin was associated with a higher bacteriological success rate, but the clinical success rates were similar for levofloxacin and clarithomycin.

Acute exacerbations of COPD characterise the natural history of COPD and are related to a decline in lung function [5], deterioration of quality of life [3] and increased costs [4]. Since $\sim 50 \%$ of all exacerbations are attributable to microbial infection [27], adequate antibiotic treatment has been shown to have beneficial effects on clinical outcome and the

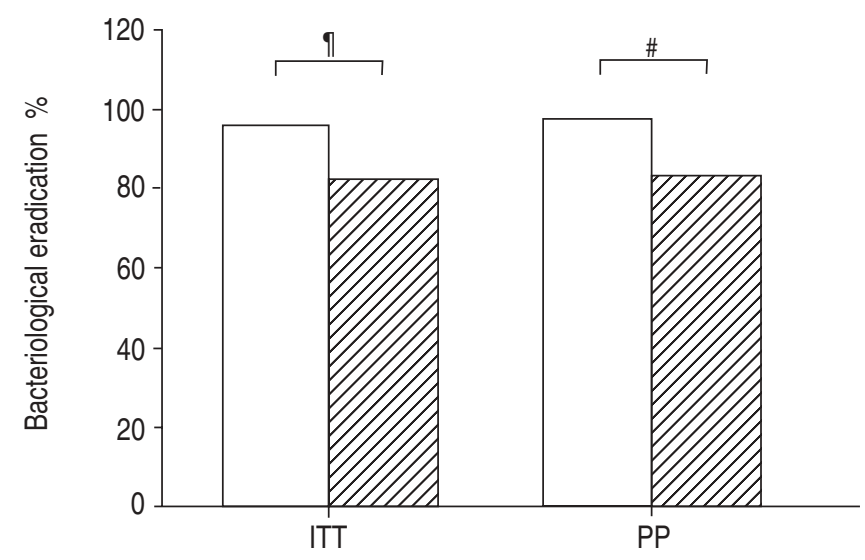

Fig. 4.-Bacteriological eradication at the end of therapy in the evaluable intent-to-treat (ITT) and per protocol (PP) populations of patients with chronic obstructive pulmonary disease exacerbation treated with levofloxacin $(\square)$ or clarithromycin $(\mathbb{\Xi})$. ${ }^{\#}$ : $\mathrm{p}=0.01 ;{ }^{\uparrow}: \mathrm{p}<0.0001$. recurrence of acute exacerbations of chronic bronchitis [22, 28].

The choice of empirical therapy has been facilitated by classification of the acute exacerbation and the related microbial spectrum according to the severity of bronchial obstruction, recurrence of annual exacerbations and comorbid conditions [29]. Despite the constant emergence of resistance to antibiotics by $H$. influenzae and $S$. pneumoniae, recent studies report similar success rates for macrolides and fluoroquinolones $[14,16,30]$, which are widely used in COPD exacerbation and also recommended by recent guidelines [31].

The EFI is a parameter that can make a difference when choosing antibiotic therapy, since fewer recurrences also mean a decrease in healthcare utilisation in COPD exacerbation. Destache et al. [32] reported in 1999 that patients with acute exacerbation of chronic bronchitis receiving ciprofloxacin, azithromycin or amoxicillin-clavulanate experience fewer hospitalisations and recurrences compared to those receiving older antibiotics.

A study by WILSON et al. [16] compared the long-term clinical outcome in patients with acute exacerbation of chronic bronchitis treated with gemifloxacin and clarithromycin and followed for a 26-week period. More patients receiving gemifloxacin remained free of recurrences compared to those receiving clarithromycin ( 71.0 versus $58.5 \%, \mathrm{p}=0.016$ ).

Another recent study comparing moxifloxacin and standard antibiotic therapy (amoxicillin, clarithromycin or cefuroxime axetil) in acute exacerbation of chronic bronchitis also found that moxifloxacin was superior in terms of clinical cure, bacteriological eradication and EFI (within 9 months of follow-up) [33]. However, this study did not differentiate between the three standard comparators, and so it is difficult to conclude whether or not moxifloxacin is really superior to each of them [34].

In contrast, no significant differences were found between levofloxacin and clarithromycin regarding EFI over the 1-yr follow-up in the present study.

This discrepancy compared to the studies of WILSON and coworkers $[16,33]$ could presumably be accounted for by the different follow-up periods used in the two studies: a period of $<1 \mathrm{yr}$ could be confounding, especially for infrequent exacerbators $\left(<2\right.$ exacerbations $\left.\cdot \mathrm{yr}^{-1}\right)$, and could result in a winter season being skipped. Another explanation may be the different nature of the exacerbation, namely whether it is due to a colonising pathogen [35] or a newly acquired microbe [6]. In the first situation, the more effective bacteriological eradication demonstrated by the quinolones may genuinely be responsible for a longer stable-state period. However, the EFI is difficult to define only in terms of successful bacteriological eradication, since a considerable number of exacerbations can be caused by a new strain, as SETHI et al. [6] showed recently.

Another aspect that can make a difference in evaluating the EFI is the severity of the bronchial obstruction. A low FEV1 has been associated with more frequent exacerbations [36], and so this group is supposed to be a more adequate target for antibiotics that prolong the stable-state period. One limitation of the present study is that most $(\sim 75 \%)$ of the patients showed a moderate degree of bronchial obstruction, which may have contributed to the low rate of exacerbations observed over $1 \mathrm{yr}$. However, similar EFIs were noted in the two severity groups irrespective of study medication.

In the present study, the bacteriological success rate was higher for levofloxacin than for clarithromycin, in relation also to the high level of in vitro resistance to clarithromycin (one-third of the $H$. influenzae strains were resistant to clarithromycin). Moreover, the proportion of patients with microbial growth in sputum was much higher $(50 \%)$ compared to other studies [16]. The differences in microbial 
eradication among studies are also related to the pattern of resistance of the centres involved.

However, similar clinical success rates were observed for levofloxacin and clarithromycin in the present study. One explanation for the non-inferior clinical efficacy of clarithromycin despite the considerable proportion of resistant bacteria could be the anti-inflammatory effect of macrolides in general [37], which may compensate the limited antimicrobial activity. Secondly, this contrast between in vitro resistance and favourable clinical outcome may also be explained by the high penetration of macrolides in different lung compartments, including the bronchial mucosa, allowing prolonged exposure to these drugs at concentrations greater than the minimum inhibitory concentration at the infection site [38].

In conclusion, treatment with levofloxacin in acute exacerbation of chronic obstructive pulmonary disease was associated with a higher bacteriological success rate than with clarithromycin. No differences in the length of exacerbationfree interval between the two study groups were observed and the frequency of recurrences over the 1-yr follow-up was also comparable. More studies are required to clarify this aspect, because of its major implications for the course of the disease and the related costs.

\begin{abstract}
Acknowledgements. Evaluation of Therapy-Free Interval in COPD Patients Study Group members: U.W. Ballies, Kiel; E. Baumann, Munich; E. Beck, Rüdersdorf; J.C. Becker, Lübeck; A. Bisping-Arnold, Freising; H. Blaufuß, Munich; V. Brauner, Bad Soden; J. Brückner, Munich; J. Clasen, Bad Oldesloe; H.W. Fischer, Verden; R. Gebhardt, Berlin; H. Grygier, Bad Homburg; U. Harnest, Munich; H.C. Hartung, Lüdenscheid; G. Hoppe, Berlin; V. Janekovic, Frankfurt am Main; B. Kemmerich, Munich; M. Korduan, Berlin; M. Ksoll, Frankfurt am Main; J. Lehnert, Munich; H. Mauch, Berlin; D. Mernitz, Munich; H. Neppl, Celle; P. Oblinger, Munich; M. Qidan, Frankfurt am Main; H.H. Ponitz, Berlin; H. Portheine, Koblenz; B. Raack, Celle; H. Riediger, Celle; K. Roscher, Bad Soden; V. Schäfer, Frankfurt am Main; E. Scheer, Berlin; H.W. Schiware, Bremen; R. Schnorr, Berlin; H. Schriewer, Lüdenscheid; G. Semrau, Berlin; W. Stengel, Lübeck; W. Tzimas, Munich; C. Uhde, Munich; L. von Versen, Berlin; W. Weede, Munich; J. Weöres, Munich; R.W. Wepler, Ulm; and C. Witt, Berlin.
\end{abstract}

\section{References}

1. Sethi S. Infectious exacerbations of chronic bronchitis: diagnosis and management. J Antimicrob Chemother 1999; 43: Suppl. A, 97-105.

2. McCrory DC, Brown C, Gelfand SE, Bach PB. Management of acute exacerbations of COPD: a summary and appraisal of published evidence. Chest 2001; 119: 1190-1209.

3. Seemungal TAR, Donaldson GC, Paul EA, et al. Effect of exacerbation on quality of life in patients with chronic obstructive pulmonary disease. Am J Respir Crit Care Med 1998; 157: 1418-1422.

4. Connors AF, Dawson NV, Thomas C, et al. Outcomes of acute exacerbations of severe chronic obstructive lung disease. Am J Respir Crit Care Med 1996; 154: 959-967.

5. Donaldson GC, Seemungal TAR, Bhowmik A, Wedzicha JA. Relationship between exacerbation frequency and lung function decline in chronic obstructive pulmonary disease. Thorax 2002; 57: 847-852.

6. Sethi S, Evans N, Grant BJ, Murphy TF. New strains of bacteria and exacerbations of chronic obstructive pulmonary disease. $N$ Engl J Med 2002; 347: 465-471.

7. Eller J, Ede A, Schaberg T, Niederman MS, Mauch H, Lode $\mathrm{H}$. Infective exacerbations of chronic bronchitis: relation between bacteriologic etiology and lung function. Chest 1998; 113: 1542-1548.

8. Murphy TF, Sethi S. Bacterial infection in chronic obstructive pulmonary disease. Am Rev Respir Dis 1992; 146: 10671083.

9. Monso E, Ruiz J, Rosell A, et al. Bacterial infection in chronic obstructive pulmonary disease. A study of stable and exacerbated outpatients using the protected specimen brush. Am J Respir Crit Care Med 1995; 152: 1316-1320.

10. Seemungal TAR, Harper-Owen R, Bhowmik A, et al. Respiratory viruses, symptoms, and inflammatory markers in acute exacerbations and stable chronic obstructive pulmonary disease. Am J Respir Crit Care Med 2001; 164: $1618-1623$

11. Rhode G, Wiethege A, Borg I, et al. Respiratory viruses in exacerbations of chronic obstructive pulmonary disease requiring hospitalisation: a case-control study. Thorax 2003; 58: 37-42.

12. Saint S, Bent S, Vittinghoff E, Grady D. Antibiotics in chronic obstructive pulmonary disease exacerbations. A meta-analysis. JAMA 1995; 273: 957-960.

13. Stockley RA, O'Brien C, Pye A, Hill SL. Relationship of sputum color to nature and outpatient management of acute exacerbations of COPD. Chest 2000; 117: 1638-1645.

14. Amsden GW, Baird IM, Simon S, Treadway G. Efficacy and safety of azithromycin vs levofloxacin in the outpatient treatment of acute bacterial exacerbations of chronic bronchitis. Chest 2003; 123: 772-777.

15. Chodosh S, McCarty J, Farkas S, et al. Randomized, double-blind study of ciprofloxacin and cefuroxime axetil for treatment of acute bacterial exacerbations of chronic bronchitis. The Bronchitis Study Group. Clin Infect Dis 1998; 27: 722-729.

16. Wilson R, Schentag JJ, Ball P, Mandell L. A comparison of gemifloxacin and clarithromycin in acute exacerbations of chronic bronchitis and long-term clinical outcomes. Clin Ther 2002; 24: 639-652.

17. Davies BI, Maesen FP. Clinical effectiveness of levofloxacin in patients with acute purulent exacerbations of chronic bronchitis: the relationship with in-vitro activity. $J$ Antimicrob Chemother 1999; 43: Suppl. C, 83-90.

18. Shah PM, Maesen FP, Dolmann A, Vetter N, Fiss E, Wesch $\mathrm{R}$. Levofloxacin versus cefuroxime axetil in the treatment of acute exacerbation of chronic bronchitis: results of a randomized, double-blind study. J Antimicrob Chemother 1999; 43: 529-539.

19. Wilson R, Kubin R, Ballin I, et al. Five day moxifloxacin therapy compared with 7 day clarithromycin therapy for the treatment of acute exacerbations of chronic bronchitis. J Antimicrob Chemother 1999; 44: 501-513.

20. American Thoracic Society. Standards for the diagnosis and care of patients with chronic obstructive pulmonary disease. Am Rev Respir Dis 1995; 152: S78-S83.

21. Lode H. Respiratory tract infections: when is antibiotic therapy indicated? Clin Ther 1991; 13: 149-156.

22. Anthonisen NR, Manfreda J, Warren CP, et al. Antibiotic therapy in acute exacerbations of chronic obstructive pulmonary disease. Ann Intern Med 1987; 106: 196-204.

23. Chodosh S, Schreurs A, Siami G, et al. Efficacy of oral ciprofloxacin vs. clarithromycin for treatment of acute bacterial exacerbations of chronic bronchitis. The Bronchitis Study Group. Clin Infect Dis 1998; 27: 730-738.

24. Bartlett JG, Ryan KJ, Smith TF, Wilson WR. Laboratory diagnosis of lower respiratory tract infections. In: Washington JA, ed. Cumitech 7A. Washington, DC, American Society for Microbiology, 1987; pp. 1-18.

25. Isenberg HD, Baron EJ, Damato RF, et al. Recommendations for the isolation of bacteria from clinical specimens. 
In: Balows A, Hausler WJ Jr, Hermann KL, et al., eds. Manual of Clinical Microbiology. Washington, American Society for Microbiology, 1991; pp. 216-237.

26. National Committee for Clinical Laboratory Standards. Performance Standards for Antimicrobial Disk Susceptibility. NCCLS Document M02-A4. 4th Edn. Villanova, NCCLS, 1990.

27. Sethi S. Infectious etiology of acute exacerbations of chronic bronchitis. Chest 2000; 117: 5: Suppl. 2, 380S-385S.

28. Adams SG, Melo J, Luther M, Anzueto A. Antibiotics are associated with lower relapse rates in outpatients with acute exacerbations of COPD. Chest 2000; 117: 1345-1352.

29. Niederman MS. Antibiotic therapy of exacerbations of chronic bronchitis. Semin Respir Infect 2000; 15: 59-70.

30. DeAbate CA, Mathew CP, Warner JH, Heyd A, Church D. The safety and efficacy of short course (5-day) moxifloxacin $v s$. azithromycin in the treatment of patients with acute exacerbation of chronic bronchitis. Respir Med 2000; 94: $1029-1037$.

31. Alvarez F, Bouza E, Garcia-Rodriguez JA, et al. Second consensus report on the use of antimicrobial agents in exacerbations of chronic obstructive pulmonary disease. Arch Bronconeumol 2003; 39: 274-283.

32. Destache CJ, Dewan N, O'Donohue WJ, Campbell JC,
Angelillo VA. Clinical and economic considerations in the treatment of acute exacerbations of chronic bronchitis. J Antimicrob Chemother 1999; 43: Suppl. A, 107-113.

33. Wilson R, Allegra L, Huchon G, et al. Short-term and longterm outcomes of moxifloxacin compared to standard antibiotic treatment in acute exacerbation of chronic bronchitis. Chest 2004; 125: 953-964.

34. Miravitlles M, Torres A. No more equivalence trials for antibiotics in exacerbations of COPD, please. Chest 2004; 125: 811-813.

35. Patel IS, Seemungal TA, Wilks M, Lloyd-Owen SJ, Donaldson GC, Wedzicha JA. Relationship between bacterial colonisation and the frequency, character and severity of COPD exacerbations. Thorax 2002; 57: 759764.

36. Donaldson GC, Seemungal TA, Patel IS, Lloyd-Owen SJ, Wilkinson TM, Wedzicha JA. Longitudinal changes in the nature, severity and frequency of COPD exacerbations. Eur Respir J 2003; 22: 931-936.

37. Culic O, Erakovic V, Parnham MJ. Anti-inflammatory effects of macrolide antibiotics. Eur J Pharmacol 2001; 429: 209-229.

38. Rodvold KA. Clinical pharmacokinetics of clarithromycin. Clin Pharmacokinet 1999; 37: 385-398. 